\title{
AN EXPERIMENTAL STUDY ON FORECASTING USING TES PROCESSES
}

\author{
Abdullah S. Karaman \\ Tayfur Altıok \\ Industrial and Systems Engineering \\ Rutgers University \\ Piscataway, NJ 08854, U.S.A.
}

\begin{abstract}
Forecasting is of prime importance for accuracy in decision making. For data sets containing high autocorrelations, failure to account for temporal dependence will result in poor forecasting. TES (Transform-Expand-Sample) is a class of stochastic processes to model empirical autocorrelated time series and is used in Monte Carlo simulation. Its merit is to simultaneously capture both the empirical distribution function and the autocorrelation function. The transition structure of TES processes can be utilized to calculate forecasts for future periods. In this paper, we utilize phase-type random variables as the innovation density in TES model fitting methodology, and we investigate the forecasting performance of TES processes compared to traditional auto regressive integrated moving-average models. We find that TES models yield forecasts as accurate as time series models.
\end{abstract}

\section{INTRODUCTION}

Improving decision making practices in a supply chain is a major source of competitive advantage in today's uncertain business environments. Resolving uncertainty in early phases of the decision making process will result in better planning and accuracy of supply chain activities, and improved customer service levels, lesser inventories and lower costs. Forecasting is one of the key ingredients necessary to clear up uncertainties in the early stages of planning. It is a crucial driver for procurement, manufacturing and distribution activities in a supply chain.

Improving the quality of forecasts has been a challenging problem. Failure to account for large autocorrelations, trend, and seasonality in data sets is the ingredient contributing to lack of accuracy in forecasting. In literature, time series models such as Winters exponential smoothing, Box-Jenkins auto regressive integrated moving-average (ARIMA), and multiple regression have been used to account for these type of patterns. Likewise, TES models can be utilized to generate forecasts for correlated data sets. Melamed (1991) introduced TES processes that can be used to generate correlated events in Monte Carlo simulation.

The primary objective of time series modelling (TSM) is to study techniques and measures for drawing inferences from past data. It accounts for the fact that data points taken through time may have an underlying structure (such as autocorrelation, trend or seasonal variation) and this structure will persist over time. The approach consists of establishing mathematical models to represent the data set. Then, the models can be employed to describe and analyze the sample data, and make forecasts for the future. The main advantage of time series models is that they can handle any persistent patterns in data (Box and Jenkins 1976, Brockwell and Davis 2002).

TES is a methodology (Jagerman and Melamed 1992a, Jagerman and Melamed 1992b, Jagerman and Melamed 1994, Melamed 1991, Melamed 1993, Melamed 1997) to model empirical time series from a stationary probability law. Its merit is to capture both the empirical distribution and autocorrelation function, simultaneously. It can model a wide variety of autocorrelation functions (e.g.monotone, oscillating, alternating etc.) and is suitable for Monte Carlo simulation of autocorrelated time series. The analytical formulas of TES process provide calculation of autocorrelations as well as its transition structure. Forecasts for the future can be calculated by utilizing the known transition structure of TES processes (Jagerman and Melamed 1995).

This paper reports an experimental study in order to compare TES process forecasting to traditional Box-Jenkins ARIMA models. Similar comparative studies exist in literature. Among the recent ones, Alon, Qi, and Sadowski (2001) presents a study that compares artificial neural networks to time-series forecasting methods in predicting US aggregate retail sales. Thomakos and Guerard (2004) compare naive, ARIMA, nonparametric and transfer function models on several data sets. Zou and Yang (2004) suggest combining several time series models to get more accurate forecasts and compare them to individual methods. Our study differs from others since it exploits TES forecasting 
procedure. Jagerman and Melamed (1995) also implement the TES forecasting methodology based on the use of mixture of uniform random variables as the innovation density. This paper contains an extensive computational study of TES forecasting, and in our experiments, we exploit phase type random variables as the innovation density.

The remainder of the paper is organized as follows. Section 2 gives an overview of TSM methodology. Section 3 explains TES processes and its empirical modelling. Section 4 illustrates the TES forecasting approach. Section 5 contains a comparison study of TES forecasting to general ARIMA models. Finally, we give a brief conclusion in section 6 .

\section{TIME SERIES MODELS}

Time series models are used to draw inferences from past data. In these models, past data is analyzed in order to identify patterns recurring over time. Then, forecasts for future periods are developed based on such underlying patterns.

The applications of time series models include forecasting future values of the series, testing hypothesis, monitoring and simulation, among others. Following is a formal definition and properties of a time series.

A discrete time series $\left\{X_{t}\right\}, t=0,1,2, \ldots$ is a sequence of observations recorded at time $t$. Correspondingly, a continuous time series is the one where observations recorded continuously. The autocorrelation function of $\left\{X_{t}\right\}$ with common mean $\mu$ and variance $\sigma^{2}<\infty$ is defined at lag $\tau$ as

$$
\rho_{x}(\tau)=\frac{E\left[\left(X_{t+\tau}-\mu\right)\left(X_{t}-\mu\right)\right]}{\sigma^{2}}, \quad \tau=1,2,3, \ldots
$$

A time series is said to be stationary, if its statistical properties remain constant over time, i.e., its mean is independent of time and its autocorrelation function is independent of time for each lag.

The autocorrelation function provides valuable information about how much successive values in a time series depends on each other. It can be thought of an indication of change in one observation if there is a change in the other. In addition, it plays an important role in forecasting future values based on the present and past values.

Box and Jenkins (1976) provides a methodology for fitting a model to an empirical data set. The systematic approach identifies a class of models appropriate for empirical data sequence at hand and estimates its parameters. A general class of Box and Jenkins models include ARIMA models that can model a large class of autocorrelation functions (Box and Jenkins 1976, Brockwell and Davis 2002). The model is a combination of auto regressive (AR) and moving-average (MA) models for differenced data. An AR model is simply a regression of the current observation to the previous ones. Formally, if $\left\{X_{t}\right\}, t=0,1,2, \ldots$ are the values of observations recorded at time $t$, then

$$
X_{t}=\phi_{1} X_{t-1}+\phi_{2} X_{t-2}+\ldots+\phi_{p} X_{t-p}+Z_{t}
$$

is called an $A R$ process of order $p$ where $Z_{t}$ is a white noise process with mean 0 and variance $\sigma^{2}$ and $\phi$ 's are finite weight parameters. On the other hand, an MA model is a regression of the current value against the previous white noise, i.e.,

$$
X_{t}=Z_{t}+\theta_{1} Z_{t-1}+\ldots+\theta_{q} Z_{t-q}
$$

where $\theta$ 's are constants. Then, $\left\{X_{t}\right\}$ is an $\operatorname{ARMA}(\mathrm{p}, \mathrm{q})$ process, if $\left\{X_{t}\right\}$ is stationary and if for every $t$,

$X_{t}-\phi_{1} X_{t-1}-\ldots-\phi_{p} X_{t-p}=Z_{t}+\theta_{1} Z_{t-1}+\ldots+\theta_{q} Z_{t-q}$.

The process $\left\{X_{t}\right\}$ is said to be an $\operatorname{ARMA}(\mathrm{p}, \mathrm{q})$ process with mean $\mu$, if $\left\{X_{t}-\mu\right\}$, deviations from the mean, is an $\operatorname{ARMA}(p, q)$ process. Finally, the integrated ARMA model, $\operatorname{ARIMA}(\mathrm{p}, \mathrm{d}, \mathrm{q})$, is an $\operatorname{ARMA}(\mathrm{p}, \mathrm{q})$ model to the $d$ times differenced data. Differencing is a tool in order to remove trend and seasonality from the empirical data.

The general approach to time series modelling includes:

- Plotting the series and examining the data for trend, seasonal component, any outlying observations and apparent changes.

- Removing the trend and seasonal components to get stationary residuals.

- Using the second order statistics of data, fitting a model to the residuals.

- Checking for goodness of fit.

- Using the model for further analysis, e.g., forecasting.

\section{TES PROCESSES}

In this section, we will give a brief overview of TES processes. TES is a modelling methodology (Jagerman and Melamed 1992a, Jagerman and Melamed 1992b, Jagerman and Melamed 1994, Melamed 1991, Melamed 1993, Melamed 1997) of empirical time series to capture its marginal distribution as well as the autocorrelation function. Together with Monte Carlo simulation, its analytical background makes it a viable tool to forecast future values of empirical time series data (Jagerman and Melamed 1995).

The TES modelling procedure satisfies three important requirements of fitting a model to an empirical data set. The first one is to match the marginal distribution of the model to the marginal distribution of the time series, which is a first-order characteristic of the data. The second one is to approximate the autocorrelation function of the data, a second-order statistics. Finally, the third requirement is 
that the sample paths generated by the TES model should resemble their empirical counterparts.

Similar to other autocorrelated sequence generation procedures, TES process utilizes background and foreground schemes. That is, an auxiliary sequence is generated from a stationary process by some recursive relationship. Then, the target foreground sequence is obtained by making a transformation of the background sequence.

There are mainly two types of TES processes, $\mathrm{TES}^{+}$and TES $^{-}$. The former is to generate positive autocorrelations while the latter is for alternating values. $\mathrm{A} \mathrm{TES}^{+}$process can be generated as follows. First, a background variate $U_{n}^{+}$is generated by utilizing the following recursive relationship:

$$
U_{n}^{+}=\left\langle U_{n-1}^{+}+V_{n}\right\rangle, \quad n>0
$$

where $U_{0}$ is a uniform random variate on $(0,1), V_{n}$ is an i.i.d random sequence (called as the innovation sequence since they bring new randomness at each step) with a common density function, $f_{v}$, independent of $U_{0}^{+}, \ldots U_{n-1}^{+}$, and \langle\rangle is the modulo- 1 arithmetic operator, i.e., $\langle x\rangle=$ $x-\max$ \{integer $n: n \leq x\}$, resulting in the fractional part of $n$. Then, the foreground sequence $X_{n}^{+}$is obtained by some transformation (called distortion) from $[0,1]$ to real numbers, i.e.,

$$
X_{n}^{+}=D\left(U_{n}^{+}\right), \quad n>0 .
$$

In order to prevent visual discontinuities in Monte Carlo sample paths generated by the TES model, an intermediary stitching transformation is applied to the background sequence. It is a piecewise linear transformation and it preserves all the properties of the original sequence. The transformation is given by

$$
S_{\xi}(y)= \begin{cases}\frac{y}{\xi}, & 0 \leq y<\xi \\ \frac{1-y}{1-\xi}, & \xi \leq y<1\end{cases}
$$

where $\xi \in[0,1)$.

Empirical TES modelling methodology consists of selecting the TES model sign, $\mathrm{TES}^{+}$or $\mathrm{TES}^{-}$, a stitching parameter, $\xi$, an inversion transformation, $D$, and an innovation density, $f_{v}$.

Initially, TES model sign is selected by investigating the empirical autocorrelations. Then, in order to specify a distortion, $D$, data sequence is modelled as an empirical histogram since any general density function can be approximated by a mixture of uniform distributions (Altiok and Melamed 2001). Consequently, $D$ is chosen as the inverse of the histogram distribution (it is composed of a transformation and a stitching function). So, the random sequence $X_{n}^{+}$has the same marginal distribution as the histogram (by the inversion transformation method (Altiok and Melamed 2001; Bratley, Fox, and Schrage 1987; Law and Kelton 1991)). Finally, a general density function, $f_{v}$, is used as the innovation density. Among the candidate densities are mixture of uniform innovations and phasetype distributions because of their generality and versatility (Alt1ok 1997). Selecting an appropriate stitching parameter and innovation density require an extensive search procedure that give rise to TES models whose autocorrelation function approximates its empirical counterpart. However, using phase-type random variables as the innovation density substantially reduces our search space since they have less parameters than mixtures of uniform random variables.

Successful applications of the TES models consist of machine fault arrivals, some financial time series models, MPEG-compressed VBR video, texture synthesis, and H.261-Compressed video (Hill and Melamed 1995a, Hill and Melamed 1995b). An algorithmic empirical TES model fitting methodology using mixture of uniform innovation sequences can be found in (Alt1ok and Melamed 2001, Jelenkovic and Melamed 1995).

\section{FORECASTING CAPABILITIES OF TES PROCESSES}

After we fit a TES model to an empirical data set using phase-type innovation variables, all the autocorrelations and transition densities of the model can be calculated using accurate analytical formulas. Utilizing the transition structure of the TES model, forecasts for future periods can be calculated as conditional expectations of the process given a current value. All the mathematical background for point estimates can be found in (Jagerman and Melamed 1995).

We employed TES forecasting methodology on various data sets. In the following figures is the implementation of the TES forecasting procedure on Dow Jones Utilities (DJU) Index, recorded between Aug.28-Dec.18, 1972 (Brockwell and Davis 2002, Makridakis and Wheelwright 1989). The TES model was constructed by matching the empirical distribution and autocorrelation function to the above mentioned data set, simultaneously. We have used phase type random variables in the construction of the TES process.

The DJU Index data set has 78 points. We split the data into fit and test periods in order to check the accuracy of the forecasting method rigorously. The TES model was identified by using 68 of them. The rest 10 points were used for out-of-sample evaluation of the forecasting approaches. In addition, we illustrated the goodness of fit of the TES model to the data in the in-sample period.

The DJU Index data can be seen in Figure 1. In addition, we have included a Monte Carlo sample path generated using the fitted TES model. It can be seen from the figure that the sample path generated by the TES model resembles its empirical counterpart which may be considered as satisfying the qualitative modelling requirement. 


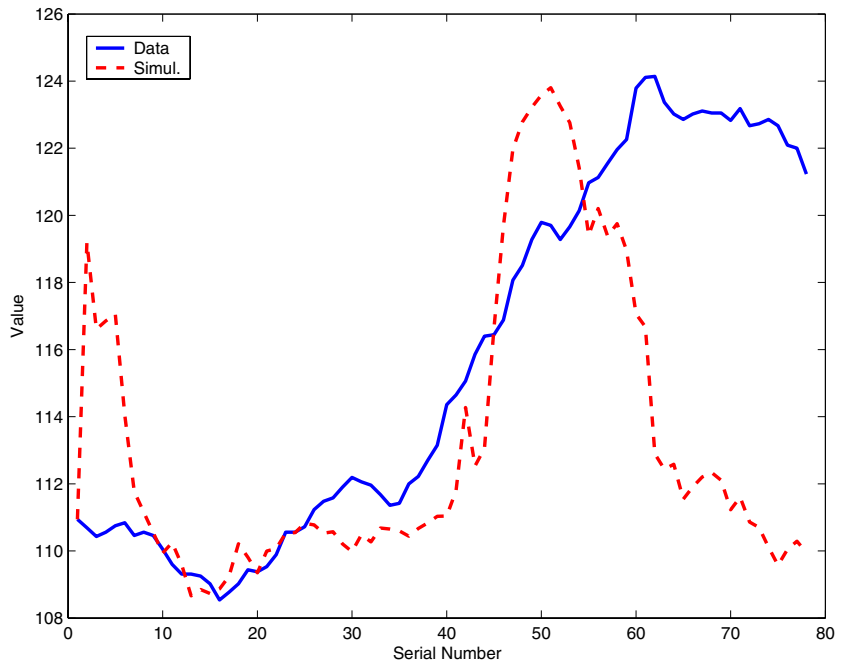

Figure 1: Empirical Data and the Generated Monte Carlo Sample Path

Further, in Figure 2, we can see the almost exact match between the autocorrelation functions of the empirical observations and the TES model.

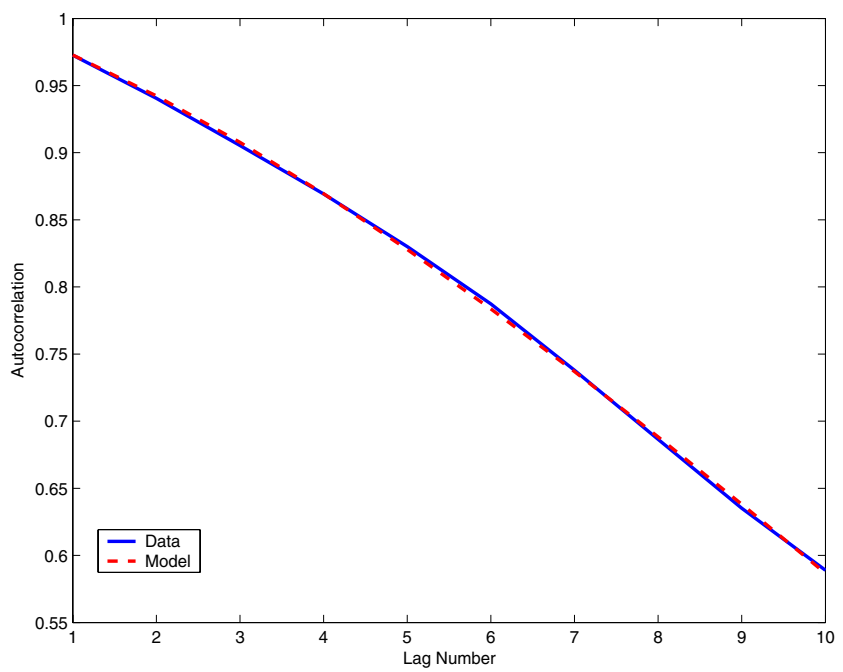

Figure 2: Autocorrelation Functions for Data and the TES Model

In the following figures, the forecasts generated by the TES model are presented. The forecasts are calculated based on the conditional expectations given the current value of the series. Therefore, for a given data point, both oneperiod-ahead and multiple-period-ahead predictions can be computed. In order to show the goodness of fit of the TES model to the in-sample data, we started conditioning on the first data point and compute estimate for the second period, conditioned on the second data point and calculate prediction for the third period, and continue in this manner till the end. In Figure 3, the graph of data and the one-period-ahead forecasts at every fit point are presented.

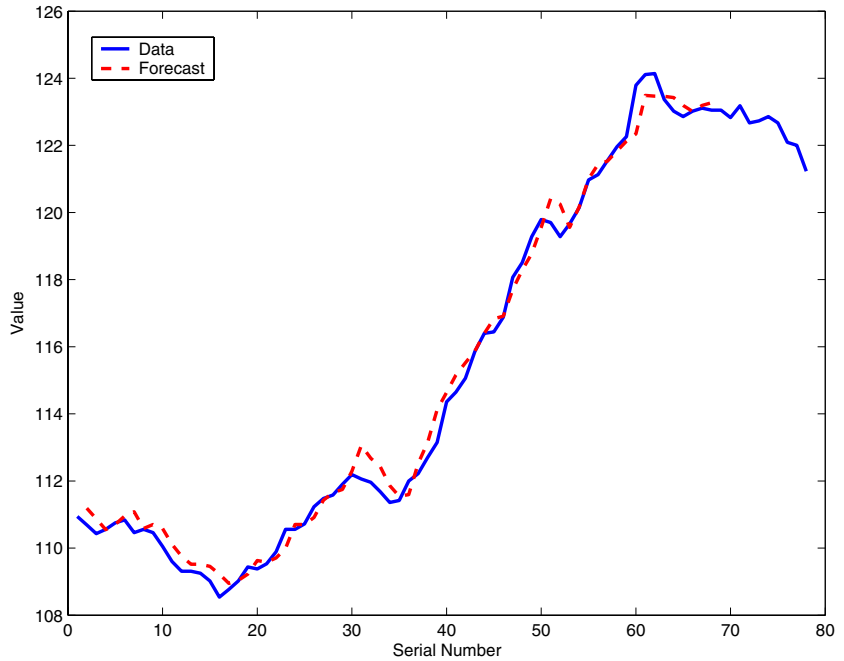

Figure 3: In-sample Forecasting for Dow Jones Utilities Index

In addition, we have illustrated the accuracy of oneperiod-ahead forecasts in the test period. In Figure 4, we have forecasted only at the out-of-sample period. For forecasting performance, we have utilized the last 10 points of the data and calculate quantities such as root mean squared error (MSE) and mean absolute percentage error (MAPE). The resulting root MSE and MAPE for out-of-sample data are 0.4561 and $\% .29$, respectively for Dow Jones Utilities Index. A comparison of this result to traditional ARIMA models can be found next section.

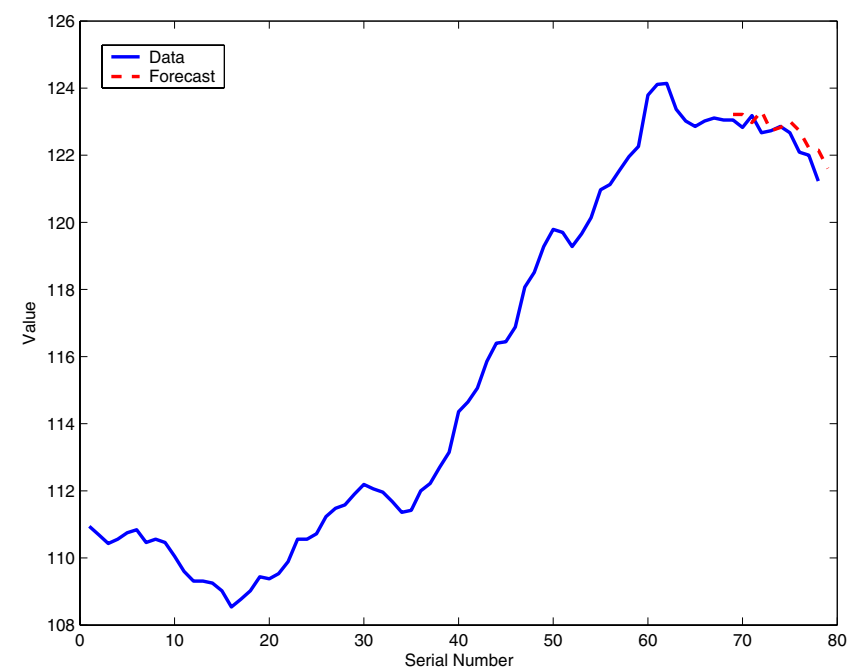

Figure 4: Out-of-sample Forecasting for Dow Jones Utilities Index

\section{COMPARISON OF TES FORECASTING TO ARIMA MODELS}

In this section, we compare the accuracy of the TES forecasting methodology to the traditional ARIMA models. The error measures of interest are square root of MSE (RMSE) 
and MAPE. The forecasting error is obtained by subtracting the forecasted values from the true values, i.e., $e_{t}=X_{t}-F_{t}$. We can calculate MSE by squaring each of the individual errors, $e_{t}$, and taking the average of those squared numbers, i.e.,

$$
M S E=\frac{\sum_{i=1}^{n} e_{i}^{2}}{n} .
$$

On the other hand, MAPE is computed by dividing the absolute errors by the corresponding true values, then averaging those deviations and multiplying by 100 , i.e.,

$$
M A P E=\frac{\sum_{i=1}^{n}\left|X_{t}-F_{t}\right| / X_{t}}{n} 100 .
$$

We have conducted extensive computational experiments to test the accuracy of TES forecasting using several empirical data sets. We have first utilized the TES modelling methodology to fit a model to an empirical data set and then generated forecasts from those fitted models. Then, we have exploited time series modelling approach to fit ARIMA models on the same data set, generated forecasts, and finally, compared the above mentioned forecasting accuracy measures, i.e., RMSE and MAPE.

Most of the data sets are borrowed from (Brockwell and Davis 2002) and for all of them, we have specified part of the data except the last ten points as the fit period and the remaining as the test points. After fitting the models, the accuracy measures were calculated using the test points.

The "Lake Huron" data shows the water level of Lake Huron in feet (reduced by 570), between 1875-1972 (Brockwell and Davis 2002, Makridakis and Wheelwright 1989). Data set "Sales" is sales data from Box and Jenkins (1976) and "Appc" represents private housing units started in the U.S.A. (monthly, from the Makridakis competition, series 922). Data set "Petroleum" is from Monthly Energy Review database and represents monthly total domestic field petroleum production from January 1984 to December 2003 (URL: <http: / / www . eia. doe.gov/emeu / mer/petro.html>). All the computations were conducted by using Matlab Release 12.1. Matlab Optimization Toolbox was utilized to fit the TES models.

Table 1 shows the computational results for different forecasting methods. In the first row, we used the Dow Jones data to compare the two forecasting procedures. TES forecasting procedure yields root mean square error of 0.4561 and mean absolute percentage error of \%0.29. In the meantime, ARIMA model yields RMSE of 0.5298 and MAPE of $\% 0.28$. As can be seen from the computational results, TES forecasting methodology yields forecasts as accurate as ARIMA models. This makes TES forecasting procedure an attractive complement to time series models, especially when data exhibits high autocorrelations.
In addition, TES processes were extremely useful in modelling empirical data series, especially in capturing autocorrelations.

\section{CONCLUSIONS}

In this paper we compared the forecasting performance of TES models to traditional time series models on several data sequence. The data sets included were highly autocorrelated which is appropriate for TES modelling. Our preliminary analysis suggests that in addition to its analytical modelling of autocorrelated time series and Monte Carlo simulation, using TES models as a forecasting tool yields forecasts as accurate as time series models. In addition, using phasetype random variables as the innovation density considerably decreases search for model fitting, which makes it possible to frequently update the fitted model.

Extension of our work include exploiting TES forecasting procedure on non-stationary time series, i.e.; when there is strong trend and seasonal patterns in time series, and determining rules for model updating procedures.

\section{ACKNOWLEDGMENTS}

We are grateful to D.L Jagerman and B. Melamed for their valuable comments and discussions on the topic.

\section{REFERENCES}

Alon, I., M. Qi, and R. J. Sadowski. 2001. Forecasting aggregate retail sales: a comparison of artificial neural networks and traditional methods. Journal of Retailing and Consumer Services 8:147-156.

Alt1ok, T. 1997. Performance analysis of manufacturing systems. New York: Springer.

Alt1ok, T., and B. Melamed. 2001. Simulation modelling and analysis with arena. New Jersey: Cyber Research, Inc.

Box, G. E. P., and G. M. Jenkins. 1976. Time series analysis: Forecasting and control. San Francisco: Holden-day.

Bratley, P., B. L. Fox, and L. Schrage. 1987. A guide to simulation. New York: Springer-Verlag.

Brockwell, P. J., and R. A. Davis. 2002. Introduction to time series and forecasting. New York: Springer.

Hill, J. R., and B. Melamed. 1995a. TEStool: A visual interactive environment for modeling autocorrelated time series. Performance Evaluation 24 (1): 3-22.

Hill, J. R., and B. Melamed. 1995b. A survey of TES modeling applications. Simulation 64 (6): 353-370.

Jagerman, D. L., and B. Melamed. 1992a. The transition and autocorrelation structure of TES processes part I: General theory. Stochastic Models 8 (2): 193-219. 
Table 1: Square Root of MSE and MAPE for TES and ARIMA

Models

\begin{tabular}{cccccc}
\hline \hline DATA SET & \multicolumn{2}{c}{ TES } & \multicolumn{3}{c}{ ARIMA } \\
\hline \hline RMSE & MAPE & RMSE & MAPE & MODEL \\
\hline Dake Huron & 0.4712 & $\% 0.29$ & 0.4366 & $\% 0.29$ & $(1,1,0)$ \\
Sales & 0.7391 & $\% 7.6$ & 0.7466 & $\% 7.6$ & $(1,0,1)$ \\
Appc & 0.8487 & $\% 0.26$ & 0.9861 & $\% 0.31$ & $(1,1,1)$ \\
Petroleum & 148.51 & $\% 6.94$ & 169.89 & $\% 7.42$ & $(1,1,3)$ \\
\hline \hline
\end{tabular}

Jagerman, D. L., and B. Melamed. 1992b. The transition and autocorrelation structure of TES processes part II: Special cases. Stochastic Models 8 (3): 499-527.

Jagerman, D. L., and B. Melamed. 1994. The spectral structure of TES processes. Stochastic Models 10 (3): 599-618.

Jagerman, D. L., and B. Melamed. 1995. Bidirectional estimation and confidence regions for TES processes. In Proceedings of MASCOTS '95, 94-98. Durham, North Carolina: IEEE Computer Society.

Jelenkovic, P., and B. Melamed. 1995. Automated TES modeling of compresses video. In Proceedings of IEEE INFOCOM '95, 746-752. Boston, Massachusetts: IEEE Computer Society.

Law, A. M., and W. D. Kelton. 1991. Simulation modeling \& analysis. New York: McGraw-Hill, Inc.

Makridakis, S., and S. C. Wheelwright. 1989. Forecasting methods for management. New York: John Wiley \& Sons.

Melamed, B. 1991. TES: A class of methods for generating autocorrelated uniform variates. ORSA J. on Computing 3:317-329.

Melamed, B. 1993. An overview of TES processes and modeling methodology. In Performance Evaluation of Computer and Communications Systems, ed. L. Donatiello and R. Nelson, 359-393.

Melamed, B. 1997. The empirical TES methodology: Modeling empirical time series. J. of Applied Mathematics and Stochastic Analysis 10 (4): 333-353.

Thomakos, D. D., and J. B. Guerard. 2004. Naive, ARIMA, nonparametric, transfer function and VAR models: a comparison of forecating performance. International Journal of Forecasting 20:53-67.

Zou, H., and Y. Yang. 2004. Combining time series for forecasting. International Journal of Forecasting 20:6984.

\section{AUTHOR BIOGRAPHIES}

ABDULLAH S. KARAMAN is a Ph.D student in the Department of Industrial and Systems Engineering at Rutgers University. He received his B.S. degree in mathematics from Koç University, Istanbul, Turkey and M.S. degree in industrial engineering from Bilkent University, Ankara,
Turkey. His research interests are forecasting and performance analysis of supply chains. His e-mail address is <akaraman@eden.rutgers. edu>.

TAYFUR ALTIOK is a Professor in the Department of Industrial and Systems Engineering at Rutgers University. He received his Ph.D. degree from the Department of Industrial Engineering at North Carolina State University at Raleigh. $\mathrm{He}$ is involved in mathematical and computer simulation modeling of manufacturing and distribution systems, marine port operations and distributed computing systems with an emphasis on transaction processing middleware. He has numerous publications including the two books in the areas of performance analysis of manufacturing systems and simulation. Dr. Altiok is a Fulbright Fellowship awardee and a recipient of numerous grants from the National Science Foundation, The United Nations, and NATO. His e-mail address is <altiok@rci.rutgers.edu>. 